\title{
Effects of high-frequency, high-intensity transcutaneous electrical nerve stimulation versus intravenous opioids for pain relief after hysteroscopy: a randomized controlled study
}

\author{
Birgitta Platon, RNA ${ }^{1}$, Sven-Egron Thörn, MD, PhD², Clas Mannheimer, MD, PhD, Paulin Andréll, MD, PhD 2,4 \\ Department of Anesthesiology and Intensive Care Medicine, ${ }^{1}$ Sahlgrenska University Hospital/Östra; ${ }^{2}$ Institute of Clinical Sciences at the Sahlgrenska \\ Academy, University of Gothenburg; ${ }^{3}$ Multidisciplinary Pain Center Kungälv Hospital Kungälv and Institute of Medicine, Sahlgrenska Academy at the \\ University of Gothenburg, Sahlgrenska University Hospital; ${ }^{4}$ Department of Anaesthesiology and Intensive Care Medicine/Paincenter, Sahlgrenska \\ University Hospital/Östra, Gothenburg, Sweden
}

\section{Objective}

To compare the time spent in the Post-Anesthesia Care Unit (PACU) and the pain-relieving effects of treatment with high-frequency, high-intensity transcutaneous electrical nerve stimulation (TENS) or intravenous (IV) opioids after hysteroscopy.

\section{Methods}

All patients who postoperatively reported a visual analogue scale (VAS) pain score of $\geq 3$ were included in the study. TENS treatment was given with a stimulus intensity between 40 and $60 \mathrm{~mA}$ for 1 minute and repeated once if there was insufficient pain relief. In the opioid group, a fractionated dose of $5 \mathrm{mg}$ morphine was administered. If the patient reported insufficient pain relief after the assigned treatment, the patient was reassigned to the other treatment group.

\section{Results}

Seventy-four women were randomized to TENS $(n=38)$ or IV opioids $(n=36)$ for treatment. Both groups reported significant pain relief after discharge from the PACU, with a decrease of VAS scores from 5.6 to 1.4 in the TENS group $(P<0.001)$ and 5.1 to 1.3 in the opioid group $(P<0.001)$. There were no significant differences between the groups. When only the responders in both groups, i.e., patients with VAS scores of $<3$ on respectively assigned treatments, were compared, the TENS responders $(n=22)$ were found to have spent a significantly shorter time in the PACU (91 vs. 69 minutes, $P=0.013$ ) compared to the opioid responders $(n=20)$.

\section{Conclusion}

Using TENS as first line of pain relief may reduce the need for postoperative opioids. In addition, TENS appears preferable as the first line of treatment due to its association with a shorter time spent in the PACU if the patient responds to the treatment.

\section{Trial Registration}

Västra Götalandsregionen Identifier: 211261

Keywords: Hysteroscopy; Pain management; Opioids; TENS
Received: 2020.03.10. Revised: 2020.05.01. Accepted: 2020.05.28. Corresponding author: Birgitta Platon, RNA

Department of Anesthesiology and Intensive Care Medicine, Sahlgrenska University Hospital/Östra, SE-416 85 Gothenburg, Sweden E-mail: birgitta.platon@vgregion.se https://orcid.org/0000-0002-9992-9578

Articles published in Obstet Gynecol Sci are open-access, distributed under the terms of the Creative Commons Attribution Non-Commercial License (http://creativecommons. org/licenses/by-nc/3.0/) which permits unrestricted non-commercial use, distribution, and reproduction in any medium, provided the original work is properly cited.

Copyright (c) 2020 Korean Society of Obstetrics and Gynecology 


\section{Obstetrics \& Gynecology Science}

Birgitta Platon, et al. TENS for postoperative hysteroscopy pain

\section{Introduction}

Hysteroscopy is a minimally invasive intervention that can be used to diagnose and treat many intrauterine and endocervical problems. Diagnostic and operative hysteroscopy have become standard in gynecologic practice. In Sweden, approximately 5,000 hysteroscopy operations are performed every year. At Sahlgrenska University Hospital Östra, approximately 160 hysteroscopy operations are performed every year [1].

Pain is sometimes a problem after hysteroscopy [2] and conservative treatment with opioids often gives adequate pain relief [3]. However, opioids have serious side effects, such as sedation, nausea and respiratory depression [4]. Hence, the patient needs surveillance, resulting in a longer time spent in the Post-Anesthesia Care Unit (PACU) after surgery.
Furthermore, there is a serious concern regarding "the opioid epidemic" in the United States [5], where society faces an escalating problem of the use of opioids after surgery, which has led to opioid addiction and sometimes abuse in some patients. Prolonged opioid use after surgery is a common and previously underestimated problem [6]. The risk of previous opioid naive patients to end up in long-term opioid use is estimated at approximately $6 \%$ after both major and minor interventions [6], indicating that long-term opioid use might be considered as a common complication after surgery. Hence, there is an increased interest in the use of nonopioid alternatives for the treatment of postoperative pain [7].

Transcutaneous electrical nerve stimulation (TENS) is a pain treatment that delivers an electrical current through the skin. The exact mechanism of action of TENS treatment is still unknown. However, the effects of peripheral nerve stimulation has traditionally been linked to the activation of afferent

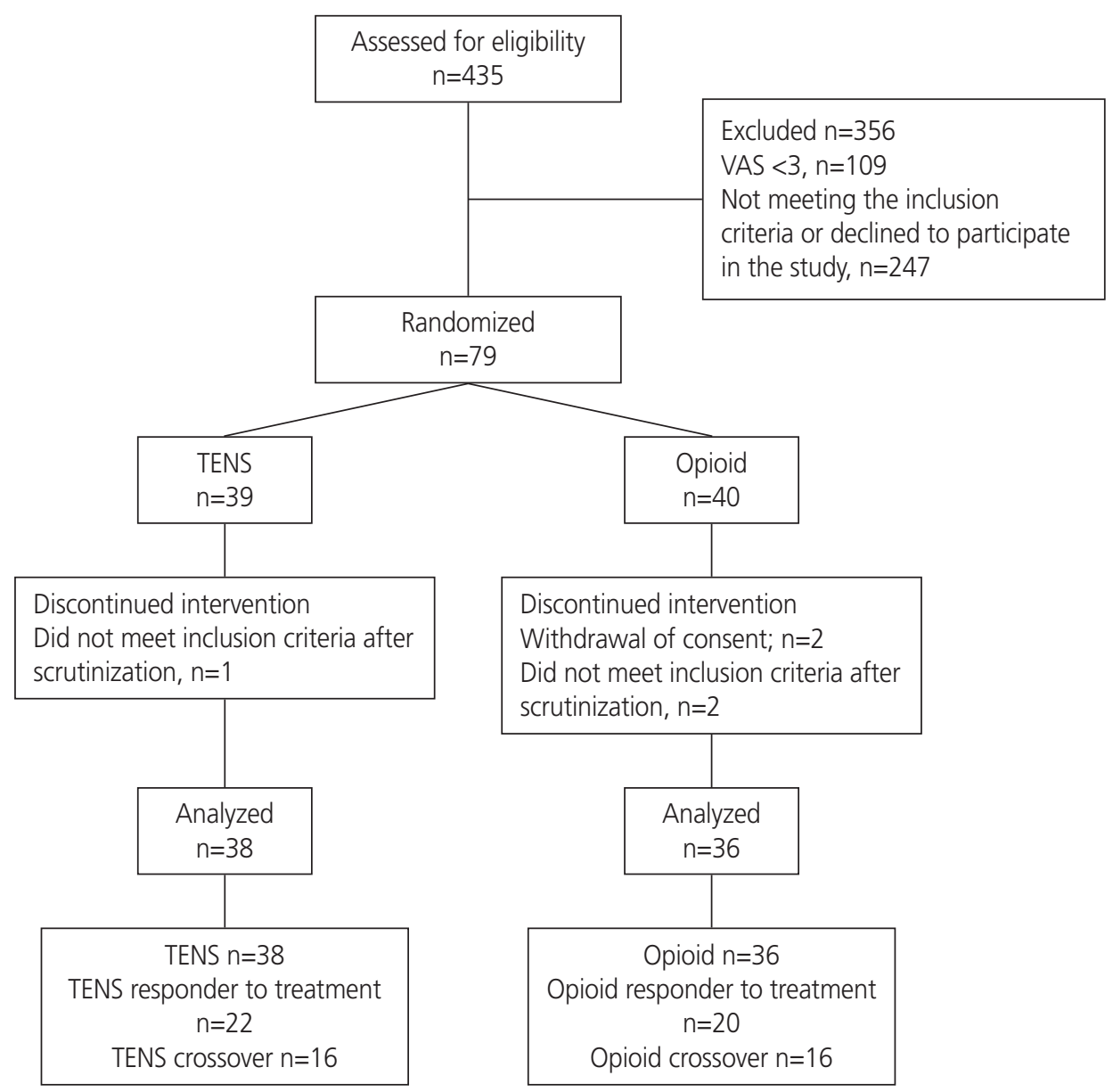

Fig. 1. Patient flowchart diagram. VAS, visual analogue scale; TENS, transcutaneous electrical nerve stimulation. 


\title{
Obstetrics \& Gynecology Science
}

\author{
Vol. 63 , No. 5, 2020
}

nerve fibers ( $A \beta$-fibers) modulating $A \delta$ and $C$-fibers in the spinal cord, which is compatible with the gate control theory of pain [8]. Release of endogenous endorphins following high-frequency TENS application has also been suggested as a mechanism of action $[9,10]$. Furthermore, the diffuse noxious inhibitory controls theory (DNIC) refers to an endogenous pain modulatory pathway, which has often been described as "pain inhibiting pain through continuous pain" by noxious or intense cutaneous stimulation, such as TENS [11].

TENS has been shown to be effective for treatment of a variety of gynecological conditions such as dysmenorrhea, pain after endometrial biopsy, surgical abortion, and gynecological laparoscopy, as well as labor pain [12-17]. In addition, previous studies from our center indicate that TENS treatment for postoperative pain results in a shorter time in the PACU $[16,17]$.

Studies by De Angelis et al. [18] and Lisón et al. [19] have indicated that TENS treatment is effective for intraoperative pain relief during hysteroscopy. However, to our knowledge, there is limited data concerning the effects of TENS for postoperative pain treatment after hysteroscopy.

Hence, the aim of the present randomized control study was to compare the time spent in the PACU after surgery as well as the postoperative pain relieving effects of high-frequency, high-intensity TENS and pharmacological treatment compared to intravenous (IV) opioids in patients undergoing hysteroscopy.

Our hypothesis was that patients receiving TENS for pain relief after undergoing hysteroscopy would spend a shorter amount of time in the PACU than those treated with pharmacological IV opioids.

\section{Materials and methods}

\section{Patients}

For 31 months, 435 patients admitted to the operating theatre at Sahlgrenska University Hospital/Östra for hysteroscopy were assessed for eligibility. Two hundred forty-seven patients declined to take part in the study, while 109 patients did not meet the inclusion criteria, i.e., pain scored $\geq 3$ on the visual analogue scale (VAS). Seventy-nine patients had VAS pain scores of $\geq 3$ i.e., requiring pain relief (Fig. 1). The exclusion criteria for the study were the following: alcohol or drug dependence, affected sensation over the current dermatome, use of a pacemaker/implantable cardioverter defibrillator (ICD), need for an interpreter, pain according to VAS of $\leq 3$, and an age less than 18 years. The patients were randomized using the closed-envelope technique after reporting a postoperative pain score $\geq 3$ according to the VAS

Table 1. Premedication, pharmacological treatment during surgery and postoperative pharmacological treatment in the Post-Anesthesia Care Unit (PACU) in the transcutaneous electrical nerve stimulation (TENS) group and in the pharmacological treatment intravenous (IV) opioid group

\begin{tabular}{lcc}
\hline Variables & TENS treatment group $(\mathbf{n = 3 8 )}$ & IV opioid treatment group $(\mathbf{n = 3 6 )}$ \\
\hline Premedication & & $31(86.1)$ \\
NSAID & $30(78.9)$ & $35(97.2)$ \\
Paracetamol & $37(97.4)$ & $36(100.0)$ \\
Pharmacological treatment during surgery & $38(100.0)$ & 0 \\
Total intravenous anesthesia & $1(2.6)$ & $36(100.0)$ \\
Fentanyl peroperatively I & & $5.3 \pm 1.9$ \\
Postoperative pharmacological treatment & $16(42.1)$ & 0 \\
Morphine IV & $3.1 \pm 5.1$ & $2(5.3)$ \\
Morphine equivalents per patient & \\
Other analgesics in the PACU & b)
\end{tabular}

All patients received morphine IV perioperatively during surgery. Data are presented as number of patients (\%), except morphine equivalents per patient, which is presented as mean \pm standard deviation.

NSAID, non-steroidal anti-inflammatory drug.

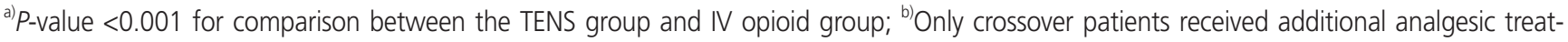
ment postoperatively. 


\section{Obstetrics \& Gynecology Science}

Birgitta Platon, et al. TENS for postoperative hysteroscopy pain

in the PACU after hysteroscopy. Both patients and investigators knew the randomization allocation. To achieve optimal pain relief, high-intensity, high-frequency TENS was used, generating paresthesia over the treated area. Consequently, it was impractical to blind both the patient and nurse to the treatment allocation.

\section{Perioperative procedures}

The standard procedure before hysteroscopy was performed according to clinical routine. All patients received a peripheral vein catheter before surgery. Preoperatively, 72 patients were administered paracetamol, while 61 patients received nonsteroidal anti-inflammatory drugs (NSAIDs) orally (Table 1). Preoperatively, the patients were administered $5 \mathrm{mg}$ morphine IV (range 2-8 $\mathrm{mg}$ ) during general propofol-remifentanil anesthesia administration. The hysteroscopy procedure was performed according to the hospital's treatment routines depending on an indication for the surgery.

\section{Postoperative procedures}

This study uses the same method as previously presented in studies from our center $[16,17]$. Thus, the description of our methods partly resembles the wording in our earlier publications.

Nurses at the PACU administered both TENS treatment as well as pharmacological treatment and assessed pain intensity according to VAS upon arrival and departure of the patients, as well as at certain time intervals during the patients' PACU stay according to clinical routine. Pain intensity was defined as severe pain with a VAS score of 7-10, moderate pain with a VAS score of 5-6, and mild pain with a VAS score of $<5$ [20]. In the TENS group, the electrodes were positioned over the area where the patient experienced pain, which was most frequently over dermatome TH12 (Fig. 2), with highfrequency $\left(80 \mathrm{~Hz}\right.$ ) stimulation (CefarPrimo ${ }^{\circledR}$, Cefar Medical $A B$, Lund, Sweden). High-intensity stimulation (strong stimulation) was achieved by increasing the stimulation gradually to achieve a stimulation intensity of 40-60 mA. Five patients found the stimulation unbearable. Thus, for these patients, only a level of 19-38 mA was achieved. The intensity of the pain was assessed directly after TENS-stimulation, i.e., after 60 seconds. If adequate pain relief was not obtained (VAS, $<3)$, the stimulation was repeated. If the patient did not experience any pain relief after 2 TENS stimulation episodes or if the patient was not able to tolerate the intensity of the stimulation, the patient received pharmacological treatment with IV opioids.

In the group given pharmacological treatment with IV opioids, a fractionated dose of an average of $5.3 \mathrm{mg}$ (range, 5-15 mg) morphine was given. The pain intensity was reestimated after approximately 10 minutes after injection. If the patient reported insufficient pain relief $(V A S,>3)$ after approximately 10 minutes, primary analgesics (i.e., morphine, paracetamol, NSAID, or tramadol) were administrated according to local clinical guidelines. If the patient still reported inadequate pain relief after 30 minutes from the start of pain treatment, TENS was administered.

A nurse assessed all patients for nausea and sedation in the PACU. Nausea was assessed using VAS (0-10; $0=a b s e n c e$ of nausea and $10=$ worst nausea). Sedation was estimated by the Ramsay sedation score [21]. The patients were transported to the ward when they were considered stable with regards to respiration and circulation (according to standard clinical criteria), and when adequate pain relief, defined as a VAS score of $<3$, had been obtained. The time of the arrival and discharge of the patient in the PACU was registered in the patients' charts. Since it is mandatory that the patients report a VAS score $<3$ before leaving the PACU, the time to obtain adequate pain relief was of interest. Hence, the time in the PACU is used in the present study as a surrogate measure for the effectiveness of the pain treatment. Other criteria for transport to the ward from the PACU were the presence of stable respiration and circulation, absence of

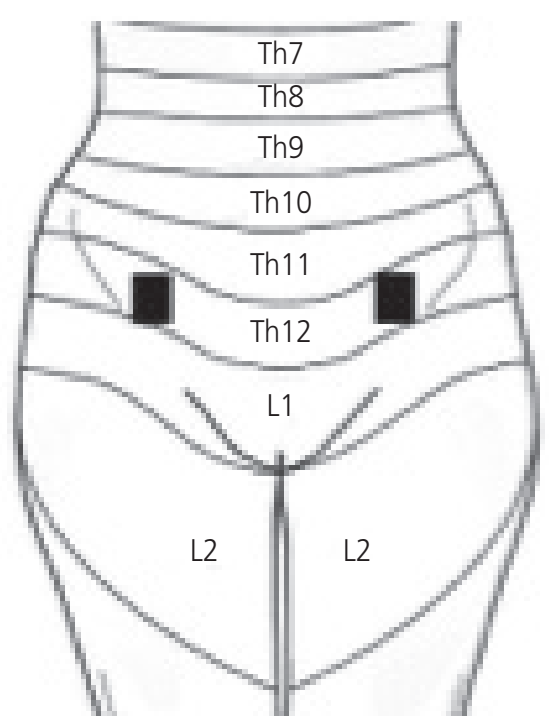

Fig. 2. Electrode placement L1 (front) and Th12 (back). 


\title{
Obstetrics \& Gynecology Science
}

\author{
Vol. 63, No. 5, 2020
}

current bleeding and nausea, and a patient who was awake and oriented to time and room.

\section{Statistical analysis}

Sample size was calculated for a power of $80 \%$ with time spent in the PACU as the primary outcome variable. To demonstrate a difference between the groups with $80 \%$ power and a 2-sided significance level of alpha 0.05, a sample size of 50 participants in each group (mean time with standard deviation in the PACU in minutes, $44.3 \pm 30.7$ vs. $62.1 \pm 34.3$ in the TENS and pharmacological treatment with IV opioid group, respectively) was required [16]. For the comparison between the 2 groups, Fisher's exact test was used for dichotomous variables. The $\chi^{2}$ analysis was used for nonordered categorical variables. The Mann-Whitney U-test was used for continuous variables. For the comparison within the groups, a Wilcoxon signed rank test was used for continuous variables. All tests were 2 -tailed, and a significance level of $5 \%$ was used. The data were analyzed based on an intention-to-treat (ITT) analysis. A subgroup analysis was performed based on "responders to treatment".

\section{Results}

\section{Patients}

The patients were randomized to TENS $(n=39)$ or standard pharmacological treatment $(n=40)$. Three patients did not meet all of the inclusion criteria while two patients withdrew consent to participate in the study. Thus, 38 patients were analyzed in the TENS group and 36 patients were analyzed in the opioid treatment group (Fig. 1).

There were no significant differences concerning health characteristics between the groups (Table 2). The indications for hysteroscopy were resection (i.e., myoma and polyps), diagnostics, extractions and other indications such as removal of placental residues postpartum, searching for intrauterine devices, and sterilization (Table 3). There were no statistically significant differences with regards to indication for surgery, time for surgery, or postoperative pain at the PACU before treatment between the groups (Tables 3 and 4). Furthermore, there were no significant differences between the groups concerning the use of premedication or pharmacological treatment during surgery (Table 1).

Table 2. Health characteristics in the transcutaneous electrical nerve stimulation (TENS) group and the pharmacological treatment intravenous (IV) opioid group

\begin{tabular}{lccc}
\hline Variables & TENS treatment group $(\mathbf{n = 3 8 )}$ & IV opioid treatment group $(\mathbf{n}=\mathbf{3 6})$ & $\boldsymbol{P}$-value \\
\hline Age $(\mathrm{yr})$ & $49.8 \pm 12.9(28-76)$ & $51.1 \pm 12.6(31-78)$ & 0.725 \\
Previous abdominal surgery & $9(23.7)$ & $11(30.6)$ & 0.604 \\
Chronic pain $^{\text {a) }}$ & $9(23.7)$ & $10(27.8 \%)$ & 0.792 \\
Analgesics use $^{a}$ & $14(36.8)$ & $8(22.2)$ & 0.251
\end{tabular}

Data are presented as number of patients (\%), except age, which is presented as mean \pm standard deviation (range).

${ }^{a)}$ Continuous analgesic use in terms of non-steroidal anti-inflammatory drug and paracetamol. One patient in the opioid group used tramadol regularly due to chronic pain.

Table 3. Indications for gynecologic hysteroscopy surgery and time for surgery in the transcutaneous electrical nerve stimulation (TENS) group and the pharmacological treatment intravenous (IV) opioid group

\begin{tabular}{lcc}
\hline Variables & TENS treatment group $(\mathbf{n = 3 8 )}$ & IV opioid treatment group $(\mathbf{n = 3 6 )}$ \\
\hline Indication for surgery ${ }^{\mathrm{a})}$ & & \\
Resection & $16(42.1)$ & $14(38.9)$ \\
Diagnostics & $1(2.6)$ & $1(2.8)$ \\
Extraction & $3(7.9)$ & $4(11.1)$ \\
Others & $18(47.4)$ & $17(47.2)$ \\
Time for surgery (min) & $21.2 \pm 13.7$ & $19.9 \pm 12.5$ \\
\hline
\end{tabular}

Data are presented as number of patients (\%), except time for surgery, which is presented as mean \pm standard deviation.

${ }^{a)}$ Comparison between the TENS group and IV opioid treatment group. 


\section{Obstetrics \& Gynecology Science}

Birgitta Platon, et al. TENS for postoperative hysteroscopy pain

\section{Time in the Post-Anesthesia Care Unit}

There were no significant differences between the groups with regards to total time spent in the PACU. The TENS group spent 87 minutes in the PACU while the pharmacological treatment with IV opioid group spent 96 minutes $(P=0.249)$ (Table 4). When only the responders to the respective treatments (i.e., patients who reported VAS $<3$ on assigned treatment) were compared (per protocol analysis), the TENS responders reported a significantly shorter time spent in the PACU compared to the responders in the opioid group (Table 5).

\section{Pain and pain relief}

Pain intensity was measured upon arrival in the PACU according to Serlin et al. [20]. There were no significant differences between the groups in terms of pain intensity. Majority of patients in the study $(n=50,67.6 \%)$ reported moderate or severe pain in the PACU. Both groups reported significant pain relief, with a decrease in VAS score from 5.6 at arrival to 1.4 at departure from the PACU in the TENS group $(P<0.001)$ and a decrease in VAS score from 5.1 at arrival and 1.3 at departure from the PACU in the opioid group $(P<0.001)$. There were no significant differences between the groups. Approximately one third $(n=24)$ of the patients reported complete pain relief (VAS, 0) after treatment (Table 4).

There was no significant difference with regards to numbers of crossovers, i.e., patients who reported a VAS score of $\geq 3$ after initial treatment, between the groups (TENS group $n=16$ [42\%]; opioid group $n=16$ [44\%]) (Fig. 1). Of the 25 crossover patients (72\%) who reported moderate or severe pain at arrival in the PACU, there were no significant differences between the crossover groups (TENS vs. opioid) with regards to pain intensity. There were no differences between the crossover group ( $n=32$, i.e., non-responders) and the responders to treatment $(n=42)$ in terms of the number of patients with mild, moderate and severe pain upon arrival in the PACU. In the crossover group, 12 (38\%) of the patients reported complete pain relief (VAS, 0) after additional treatment. Nevertheless, postoperative opioid consumption was significantly lower $(P<0.001)$ in the TENS group $(3.1$ morphine equivalents per patients) versus the IV opioid group (5.3 morphine equivalents per patients) despite the presence of crossover patients (Table 1).

\section{Chronic pain}

Nineteen $(26 \%)$ of the patients in the study suffered from chronic pain (Table 2$)$, while 8 (25\%) of the patients in the crossover group had chronic pain (TENS $n=3$, opioid $n=5$ ).

Table 4. Pain relief and time in the Post-Anesthesia Care Unit (PACU) in the transcutaneous electrical nerve stimulation (TENS) group and in the pharmacological treatment intravenous (IV) opioid group according to visual analogue scale (VAS)

\begin{tabular}{lccc}
\hline Variables & TENS treatment group $(\mathbf{n = 3 8 )}$ & IV opioid treatment group $(\mathbf{n = 3 6 )}$ & $\boldsymbol{P}$-value \\
\hline VAS before treatment & $5.6 \pm 1.8$ & $5.1 \pm 1.3$ & 0.408 \\
VAS at leave from the PACU & $1.4 \pm 1.2^{\mathrm{a})}$ & $1.3 \pm 1.1^{\mathrm{a})}$ & 0.698 \\
VAS 0 after treatment & $12(31.6)$ & $12(33.3)$ & 1.000 \\
Time at the recovery ward (min) & $87.3 \pm 36.5$ & $96.4 \pm 38.5$ & 0.249 \\
\hline
\end{tabular}

Data are presented as mean \pm standard deviation, except VAS 0 after treatment, which is presented as number (\%).

a) $P$-value within groups, before treatment and at discharge from the PACU; TENS <0.0001, IV opioid $<0.0001$.

Table 5. Pain relief and time in the Post-Anesthesia Care Unit (PACU) according to visual analogue scale (VAS) in the patients who responded to transcutaneous electrical nerve stimulation (TENS) treatment and pharmacological treatment intravenous (IV) opioids (VAS, $<3)$, i.e., per protocol analysis

\begin{tabular}{lccc}
\hline Variables & TENS treatment group $(\mathbf{n = 2 2 )}$ & IV opioid treatment group $(\mathbf{n = 2 0})$ & $\boldsymbol{P}$-value \\
\hline VAS before treatment & $5.2 \pm 1.7$ & $4.8 \pm 1.2$ & 0.396 \\
VAS at leave the PACU & $1.1 \pm 1.2$ & $1.6 \pm 0.9$ & 0.082 \\
VAS O after treatment & $9(40.9)$ & $3(15.0)$ & 0.091 \\
Time in the PACU (min) & $68.8 \pm 20.1$ & $91.0 \pm 34.4$ & 0.013 \\
\hline
\end{tabular}

Data are presented as mean \pm standard deviation, except VAS 0 after treatment, which is presented as number (\%). 


\title{
Obstetrics \& Gynecology Science
}

\author{
Vol. 63, No. 5, 2020
}

\section{Nausea and sedation}

There were no significant differences with regards to sedation, nausea and use of anti-emetics between the groups (data not shown).

\section{Discussion}

The results from this randomized controlled study indicate that both treatment with high-intensity, high-frequency stimulation using TENS and pharmacological treatment with IV opioids are effective for postoperative pain relief after gynecologic hysteroscopy. This is consistent with previous studies demonstrating the effectiveness of TENS for postoperative pain relief $[16,17]$. However, there was no difference between the groups with regards to time spent in the PACU after surgery. When only responders to treatment were compared, both groups reported similar pain relief, but the TENS group spent a shorter time in the PACU.

If the patient responds to the TENS treatment, the patient reports instant pain relief with no need for monitoring of adverse side-effects such as sedation, nausea and respiratory depression after treatment. This is a clinically relevant advantage with the TENS treatment modality, since it only takes two minutes to assess if the patient responds to it or not. In contrast, it takes approximately 20 minutes to evaluate the effects of pharmacological treatment with IV opioid [22]. Thus, pharmacological treatment with IV opioids is delayed by at most a few minutes if the patient does not respond to TENS, making TENS a more preferable choice for initial pain treatment in the PACU.

Chronic pain after surgery in the lower part of the abdomen is a frequent complication, with a study showing that $5-32 \%$ of the patients develop chronic pain after hysterectomy [23]. Thus, it is important to achieve optimal postoperative pain treatment to prevent development of chronic pain. More than 2/3 of the patients reported moderate or severe pain upon arrival in the PACU. Thus, obtaining adequate pain relief was difficult for many patients, resulting in a high number of crossovers in more than $40 \%$ of the patients ( $n=32$ ) wherein they received both pharmacological treatment with IV opioid and TENS to obtain sufficient pain relief (VAS, <3). The crossover group did not differ from the responders to treatment with regards to pain intensity or previous chronic pain. Nevertheless, opioid consumption was significantly lower in the TENS group. Thus, using TENS as the first line of pain relief may reduce the need for postoperative opioids.

Previously, other methods for peri- and postoperative pain relief such as para-cervical blockade has been tested after hysteroscopy. However, these have limited effects on postoperative pain after uterus intervention [24]. Furthermore paracervical blockade entails a risk of inducing bradycardia and hypotension, which is likely a result of accidental intravascular injection [25]. Thus, TENS seems to be a more effective method for postoperative pain relief after hysteroscopy with the benefit of being completely reversible within seconds and with limited side effects.

The importance of properly managing postoperative pain treatment is well known partly because of the risk of developing chronic pain after surgery due to insufficient pain treatment [26]. Furthermore, it is important to reduce the suffering of patients and minimize any complications due to inadequate pain treatment, such as impaired respiration and immobilization.

In the pursuit of reducing or even replacing opioids, a less harmful alternative for the patient such as TENS might be considered a suitable choice due to the minimal frequency of side effects associated with short-term TENS treatment. Based on the results of this present study and the possible serious short- and long-term complications of opioid treatment, TENS may to preferable as a first line of pain relief in patients undergoing hysteroscopy reporting a postoperative pain VAS score of $\geq 3$.

In the present study, high-frequency $(80 \mathrm{~Hz})$, high-intensity (40-60 mA) TENS stimulation for 60 seconds was used, and if needed, was repeated once. This is the same treatment model used for primary dysmenorrhea, surgical abortion, gynecological laparoscopy and angina pectoris $[13,16,17,27]$. It is important to achieve a strong stimulation level (at least 40 $m A)$. By gradually increasing stimulation intensity, most patients tolerated the treatment, which is in line with previous studies $[16,17]$. Stimulation intensity seems to be crucial for the pain-relieving effect. Results from a study by Moran et al. [28] indicate that strong non-painful intensities seem more efficient than mild intensity stimulation. Furthermore, for optimum pain relief, it seems important to adjust the position of the electrodes so that the paresthesia over the abdomen covers the area in which the patient localized the abdominal pain. Before the start of the treatment, skin sensation should be assessed, since TENS should not be used if there 


\section{Obstetrics \& Gynecology Science}

Birgitta Platon, et al. TENS for postoperative hysteroscopy pain

is impaired sensation. There were no reported side effects of TENS treatment in the study except for intolerable intensities of stimulation for some patients. However, the stimulation lasted for only 60 seconds and all the women in the TENS group were informed that this short period of (uncomfortable) high-intensity TENS was needed to obtain postoperative pain relief. To acclimatize the patients, the stimulation intensity was increased gradually to obtain a high-intensity stimulation level. An additional advantage of TENS treatment is that there is no need to monitor the patients after the treatment. This is in contrast to pharmacological treatment with IV opioids, wherein patients need to be assessed due to the risk of respiratory depression associated with opioids. The clinical routine for TENS treatment at our center has been previously described in detail $[16,17]$.

In addition to the pain-relieving effect of TENS and opioids, the cost of treatment needs to be considered. The TENS device used in the present study costs 121 Euros. At our center, costs for consumable materials, i.e., the reusable electrodes and electrode gel, amounted to less than approximately 20 Euros for the study patients, i.e., 0.37 Euro per patient. In comparison, one ampoule of 10 milligrams per milliliter morphine costs 0.31 Euros. In the present study, 1 to $2 \mathrm{am}$ poules of morphine were used per patient. However, the TENS device can be used for several daily treatments for up to 10 years. Furthermore, assistant nurses can administrate the TENS treatment, whereas morphine can only be administrated by nurses (or physicians) and the patient needs to be monitored for at least 30 minutes due to side-effects, especially respiratory depression. Hence, the nurses' competence and time can be used for other specific tasks when using TENS for postoperative pain treatment. Furthermore, if the patient responded to TENS treatment, their time spent in the PACU was reduced by 22 minutes, which has an impact on the overall cost for the stay in PACU and which enables them to perform more operations per day.

First, the surgical indication for hysteroscopy affects the time and difficulty of the surgery as well as the quantity of distending media, among others, which may in turn affect postoperative pain. In the present study, there was no difference with regards to indication for hysteroscopy and time of surgery between the groups. Hence, the peri-operative conditions seem to not have affected the patients' reported postoperative pain at the PACU before treatment.

Second, it was not possible to have a control group un- treated for postoperative pain for ethical reasons. Hence, pharmacological treatment with IV opioids and TENS treatment were compared with each other. It would have been a good addition to have blinded the patients and investigators to their respective treatment allocations. However, due to technical and clinical logistic reasons, it was not possible to blind the investigators, and most importantly the patients, to the treatment allocation. This might have affected the outcome. Nevertheless, it should be emphasized that the patients reported their own experiences pain intensities using the VAS scale (i.e., the nurse did not estimate or influence the patient's report).

Third, the large number of crossovers might have affected the results.

Fourth, according to the power calculation, 100 patients should be included to demonstrate a possible statistical difference between the groups. However, due to reorganization in the hospital resulting in allocation of the patient groups to a different hospital, only 79 patients were randomized for the present study.

In general, results from clinical trials will be stronger with the same results from other independent groups. Hence, further randomized controlled studies are warranted to confirm the pain-relieving effect of TENS on postoperative pain and its effects on opioid consumption.

TENS and short-term pharmacological treatment with IV opioids are both effective treatments for pain relief after gynecologic hysteroscopy, with more than one third of patients reporting complete pain relief. However, many patients seem to need a combination of both treatments to obtain adequate pain relief. Thus, further studies are warranted to further characterize this group of patients.

TENS may be preferable as a first line of treatment since it is associated with a shorter time spent in the PACU and a faster onset of pain relief if the patient responds to the treatment compared to IV opioids. In addition, the effects of TENS treatment can be assessed in a few minutes, which allows for a prompt shift to pharmacological treatment with IV opioids if the patient does not respond to TENS treatment. Thus, TENS as a first-line pain relief method is an option to reduce the need for postoperative opioids. 


\title{
Obstetrics \& Gynecology Science
}

\author{
Vol. 63, No. 5, 2020
}

\section{Acknowledgements}

We would like to thank our medical statistician, Nils-Gunnar Pehrsson, and our nurse anesthesiologist, Cecilia Ögren, for their expert professional advice. We would also like to acknowledge the support of the patients and nurses in the Post-Anesthesia Care Unit (PACU) and the gynecologic ward who made this study possible.

The study was financed by grants from the Swedish state under the agreement between the Swedish government and the county councils, the ALF-agreement (ALFGBG-724711). The Department of Anesthesiology and Intensive Care Medicine, Sahlgrenska University Hospital/Östra supported the study.

\section{Conflict of interest}

No potential conflict of interest relevant to this article was reported.

\section{Ethical approval}

The study is in accordance with the Helsinki Declaration of 1975 as revised in 2000. The Regional Ethics Review Board in Gothenburg, Sweden approved the study (Dnr 442-13).

\section{Patient consent}

All participants received written and verbal information regarding the study before giving their written consents to participate in the study.

\section{References}

1. Zacharias F. Årsrapport hysteroskopi 2015. Från gynopregistret delregister: hysteroskopi [Internet]. Umeå: Nationella Kvalitetsregistret inom Gynekologisk Kirurgi; 2016 [cited 2018 May 15]. Available from: http://www. gynop.se/wp-content/uploads/2017/07/Gynop_Hysteros kopi_\%C3\%85rsrapport2015.pdf. Swedish.

2. Paulo AA, Solheiro MH, Paulo CO, Afreixo VM. What proportion of women refers moderate to severe pain during office hysteroscopy with a mini-hysteroscope? A systematic review and meta-analysis. Arch Gynecol Obstet 2016;293:37-46.

3. Hassan $A$, Haggag $\mathrm{H}$. Role of oral tramadol $50 \mathrm{mg}$ in reducing pain associated with outpatient hysteroscopy: a randomised double-blind placebo-controlled trial. Aust N Z J Obstet Gynaecol 2016;56:102-6.

4. Borgbjerg FM, Nielsen K, Franks J. Experimental pain stimulates respiration and attenuates morphine-induced respiratory depression: a controlled study in human volunteers. Pain 1996;64:123-8.

5. Hah JM, Bateman BT, Ratliff J, Curtin C, Sun E. Chronic opioid use after surgery: implications for perioperative management in the face of the opioid epidemic. Anesth Analg 2017; 125:1733-40.

6. Brummett CM, Waljee JF, Goesling J, Moser S, Lin P, Englesbe MJ, et al. New persistent opioid use after minor and major surgical procedures in US adults. JAMA Surg 2017;152:e170504.

7. Jafra A, Mitra S. Pain relief after ambulatory surgery: progress over the last decade. Saudi J Anaesth 2018;12:618-25.

8. Melzack R, Wall PD. Pain mechanisms: a new theory. Science 1965;150:971-9.

9. Facchinetti F, Sandrini G, Petraglia F, Alfonsi E, Nappi G, Genazzani AR. Concomitant increase in nociceptive flexion reflex threshold and plasma opioids following transcutaneous nerve stimulation. Pain 1984;19:295-303.

10. Leonard G, Goffaux P, Marchand S. Deciphering the role of endogenous opioids in high-frequency TENS using low and high doses of naloxone. Pain 2010;151:215-9.

11. Le Bars D, Dickenson AH, Besson JM. Diffuse noxious inhibitory controls (DNIC). II. Lack of effect on non-convergent neurones, supraspinal involvement and theoretical implications. Pain 1979;6:305-27.

12. Proctor ML, Smith CA, Farquhar CM, Stones RW. Transcutaneous electrical nerve stimulation and acupuncture for primary dysmenorrhoea. Cochrane Database Syst Rev 2002;(2):CD002123.

13. Milsom I, Hedner N, Mannheimer C. A comparative study of the effect of high-intensity transcutaneous nerve stimulation and oral naproxen on intrauterine pressure and menstrual pain in patients with primary dysmenorrhea. Am J Obstet Gynecol 1994;170:123-9. 


\section{Obstetrics \& Gynecology Science}

Birgitta Platon, et al. TENS for postoperative hysteroscopy pain

14. Yilmazer M, Kose S, Arioz DT, Koken G, Ozbulut O. Efficacy of transcutaneous electrical nerve stimulation for pain relief in women undergoing office endometrial biopsy. Arch Gynecol Obstet 2012;285:1059-64.

15. Kaplan B, Rabinerson D, Lurie S, Bar J, Krieser UR, Neri A. Transcutaneous electrical nerve stimulation (TENS) for adjuvant pain-relief during labor and delivery. Int J Gynaecol Obstet 1998;60:251-5.

16. Platon B, Andréll P, Raner C, Rudolph M, Dvoretsky A, Mannheimer $C$. High-frequency, high-intensity transcutaneous electrical nerve stimulation as treatment of pain after surgical abortion. Pain 2010;148:114-9.

17. Platon B, Mannheimer C, Andréll P. Effects of highfrequency, high-intensity transcutaneous electrical nerve stimulation versus intravenous opioids for pain relief after gynecologic laparoscopic surgery: a randomized controlled study. Korean J Anesthesiol 2018;71:149-56.

18. De Angelis C, Perrone G, Santoro G, Nofroni I, Zichella L. Suppression of pelvic pain during hysteroscopy with a transcutaneous electrical nerve stimulation device. Fertil Steril 2003;79:1422-7.

19. Lisón JF, Amer-Cuenca JJ, Piquer-Martí S, BenaventCaballer V, Biviá-Roig G, Marín-Buck A. Transcutaneous nerve stimulation for pain relief during office hysteroscopy: a randomized controlled trial. Obstet Gynecol 2017;129:363-70.

20. Serlin RC, Mendoza TR, Nakamura Y, Edwards KR, Cleeland CS. When is cancer pain mild, moderate or severe? Grading pain severity by its interference with function.
Pain 1995;61:277-84.

21. De Jonghe B, Cook D, Appere-De-Vecchi C, Guyatt G, Meade $M$, Outin $H$. Using and understanding sedation scoring systems: a systematic review. Intensive Care Med 2000;26:275-85.

22. Aubrun F, Mazoit JX, Riou B. Postoperative intravenous morphine titration. Br J Anaesth 2012;108:193-201.

23. Brandsborg B, Nikolajsen L. Chronic pain after hysterectomy. Curr Opin Anaesthesiol 2018;31:268-73.

24. Tangsiriwatthana T, Sangkomkamhang US, Lumbiganon P, Laopaiboon M. Paracervical local anaesthesia for cervical dilatation and uterine intervention. Cochrane Database Syst Rev 2013;(9):CD005056.

25. Lau WC, Lo WK, Tam WH, Yuen PM. Paracervical anaesthesia in outpatient hysteroscopy: a randomised doubleblind placebo-controlled trial. Br J Obstet Gynaecol 1999;106:356-9.

26. Kehlet $H$, Jensen TS, Woolf CJ. Persistent postsurgical pain: risk factors and prevention. Lancet 2006;367:161825.

27. Mannheimer C, Carlsson CA, Vedin A, Wilhelmsson C. Transcutaneous electrical nerve stimulation (TENS) in angina pectoris. Pain 1986;26:291-300.

28. Moran F, Leonard T, Hawthorne S, Hughes CM, McCrum-Gardner $\mathrm{E}$, Johnson $\mathrm{Ml}$, et al. Hypoalgesia in response to transcutaneous electrical nerve stimulation (TENS) depends on stimulation intensity. J Pain 2011;12:929-35. 